\title{
BMJ Open Education, night splinting and exercise versus usual care on recovery and conversion to surgery for people awaiting carpal tunnel surgery: a protocol for a randomised controlled trial
}

\author{
Karina J Lewis, ${ }^{1,2}$ Leo Ross, ${ }^{3}$ Michel W Coppieters, ${ }^{2,4}$ Bill Vicenzino, ${ }^{2}$ \\ Annina B Schmid ${ }^{2,5}$
}

To cite: Lewis KJ, Ross L, Coppieters MW, et al. Education, night splinting and exercise versus usual care on recovery and conversion to surgery for people awaiting carpal tunnel surgery: a protocol for a randomised controlled trial. BMJ Open 2016;6:e012053. doi:10.1136/bmjopen-2016012053

- Prepublication history and additional material is available. To view please visit the journal (http://dx.doi.org/ 10.1136/bmjopen-2016012053).

Received 25 March 2016 Revised 15 June 2016 Accepted 5 August 2016

\section{CrossMark}

For numbered affiliations see end of article.

Correspondence to A/Prof Annina Schmid; annina.schmid@ neuro-research.ch

\section{ABSTRACT}

Introduction: Carpal tunnel syndrome (CTS) is a prevalent upper limb condition that results in significant individual and socioeconomic costs. Large patient numbers, long outpatient waiting times and traditional referral pathways in public health systems create delays in accessing treatment for this condition. Alternative care pathways aimed at streamlining access to treatment and reducing the need for surgical intervention warrant further investigation.

Methods: A randomised, single-blind controlled clinical trial will be conducted. 128 participants aged 18-75 years with CTS will be recruited from the carpal tunnel surgery waitlists of participating public hospitals. Suitable participants will be stratified for severity and randomly allocated to either receive therapy (education, provision of splints and a home exercise programme) or standard care (continuing on the waitlist without hand therapy intervention for the duration of the study). Outcomes will be measured at baseline and after 6 weeks and 6 months. Primary outcomes are conversion to surgery ratio and perceived effect via the Global Rating of Change Scale.

Secondary measures include patient satisfaction, and monitoring of symptoms and function using outcome measures including the Boston CTS Questionnaire, Disability of Arm, Shoulder and Hand Questionnaire, Patient-Specific Functional Scale, patient completed diagram of symptoms and Self-reported Leeds Assessment of Neuropathic Symptoms and Signs pain scale.

Discussion: This paper outlines the design and rationale for a randomised controlled trial that aims to assess the efficacy of an alternative care pathway for the management of patients with CTS while on the surgery waitlist. It is anticipated that the outcomes of this study will contribute to improved and expedited management of this common condition in a public hospital setting.

Ethics and dissemination: Ethics approval was granted by the Princess Alexandra Hospital Centres for Health Research (HREC/13/QPAH/434-SSA/13/QPAH/ 447) and the Medical Research Ethics Committee at

\section{Strengths and limitations of this study}

The protocol describes a prospective randomised controlled trial to assess the efficacy of an alternative care pathway for the management of carpal tunnel syndrome.

- The trial uses a robust pragmatic design replicating usual clinical practice.

- The results of this study will contribute to improved and expedited management of carpal tunnel syndrome in a public hospital setting.

the University of Queensland. Results will be disseminated via conferences and peer-reviewed publications.

Trial registration number: ACTRN12613001095752.

\section{INTRODUCTION}

Carpal tunnel syndrome (CTS) is a condition caused by compression of the median nerve as it passes through the carpal tunnel at the wrist. ${ }^{1}$ CTS is the most common nerve entrapment $^{2}$ with an estimated prevalence of $3.8 \%$ in the general population, ${ }^{3}$ and $7.8 \%$ in the working population. ${ }^{4}$ Symptoms include paraesthesia, pain, weakness and loss of dexterity in the affected hand. ${ }^{5}$ Although there are several risk factors associated with CTS (such as age, diabetes and sex), in many cases, there is no identifiable causal mechanism or comorbidity. ${ }^{6}$ CTS is associated with a significant socioeconomic burden due to its impact on productivity, function, quality of life and significant costs associated with its management. ${ }^{67}$

CTS is managed either surgically or nonsurgically, with stronger evidence in support 
of surgery compared to non-surgical options. ${ }^{8}{ }^{9}$ Despite well-documented evidence regarding the significant socioeconomic impact, CTS is typically considered a low surgical priority in publicly funded health systems. ${ }^{10}$ The reality of long public hospital waiting times and traditional referral pathways (general medical practitioner to surgeon to therapist) creates significant delays in gaining access to treatment. Not only are these substantial waiting times likely to result in extended periods of reduced quality of life, ${ }^{11}$ they may also compromise longterm outcomes as delayed surgery has been shown to be associated with poorer prognosis. ${ }^{12}$

Guidelines endorsed by professional associations suggest a trial of non-surgical interventions for patients with mild or moderate CTS symptoms, with surgery being the treatment of choice where symptoms are severe or prolonged, or for those whose conservative management has been unsuccessful. ${ }^{6}{ }^{13}$ Commonly recommended non-surgical interventions include use of splints, nerve and tendon gliding exercises and activity modification. $^{5} \quad 6 \quad$ 14-16 These recommendations are largely based on clinical observations as there is limited research-based evidence to guide non-surgical treatments. Given the unconvincing evidence for conservative approaches and the resulting reliance on clinical trends to guide practice, further investigation into nonsurgical interventions is warranted.

The number of surgical interventions performed in the UK for CTS has been predicted to increase from 66833 per year in 2015 to 104922 per year by $2030 .^{17}$ Given that a single carpal tunnel release in the UK is projected to cost between $£ 830^{18}$ and $£ 2600,{ }^{19}$ the extrapolated total cost of carpal tunnel release surgery to the UK health budget will exceed $£ 55$ million in 2015 . This level of expenditure creates incentives for publicly funded health systems to manage CTS efficiently. In an effort to manage surgery waitlists and reduce costs, retrospective studies in the UK and Australia have examined the effect of alternative care pathways and therapist-led clinics for patients with CTS on surgical waitlists. ${ }^{20-22}$ Despite limited data regarding the cost or clinical effectiveness of conservative interventions, ${ }^{15}{ }^{16}$ these retrospective case audits have shown a clear reduction in CTS surgery waitlists. The potential benefits of these models of care therefore warrant further investigation.

The aim of this project is to evaluate the efficacy of an alternative care pathway compared to standard care on the need for surgery and patient-rated outcomes in the management of patients with CTS while on the surgical waitlists.

\section{METHODS/DESIGN}

A randomised controlled multisite clinical trial will be conducted in four publicly funded hospitals within Queensland, Australia. This trial will compare therapeutic intervention (education, provision of splints and a home exercise programme (ESX)) to the current standard care (continuing on the surgery waitlist without conservative intervention) in the management of patients with CTS (figure 1).

\section{Participants}

Patients with a diagnosis of CTS who are on the orthopaedic department outpatient waitlist of participating Queensland Health hospitals will be contacted by telephone. Those interested in participating in the study will be sent an information sheet outlining the study as well as a brief questionnaire to assess eligibility. Patients who meet the selection criteria (box 1) and agree to participate will be invited for a baseline assessment by an occupational therapist or physiotherapist employed within the Hand Therapy department of the participating hospitals. During this first appointment, informed written consent will be gained from each participant. This study has received ethical clearance and approval from the relevant hospital and university ethics review boards.

\section{Baseline assessment}

During the first appointment, a clinical examination will be completed. This examination will include a detailed medical and social history (occupation, sports, hobbies), demographic data (age, gender, weight, height review of the nature and onset of symptoms, and observation for thenar eminence wasting).

\section{Interventions}

Once consented and included in the study, the patients will be allocated to randomly receive standard care or a programme of ESX.

\section{Education, splinting and exercise}

ESX will be provided by either an occupational therapist or physiotherapist during a single appointment, and then continues as a patient self-applied home-based therapy programme while the patients are on the orthopaedic department outpatient waitlist.

Patients allocated to receive ESX will attend a 20 30 min group education presentation by an occupational therapist or physiotherapist on the same day as their baseline assessment. This presentation will cover education regarding the pathophysiology of CTS, treatment options (conservative management and surgery), posture and activity modification principles. ${ }^{23}$ This information will also be provided in the form of an education booklet which participants will be encouraged to review at home.

Participants will also be provided with a splint. The Berger test ${ }^{5}$ will be used to determine if lumbrical muscle excursion into the carpal tunnel may be contributing to CTS symptoms. ${ }^{24-26}$ This test involves the participant actively holding the fingers in full flexion with their wrist in neutral position. If symptoms worsen within $30 \mathrm{~s}$, the test is deemed to be positive. ${ }^{5}$ Those with a negative Berger test will receive neoprene wrist 
Figure 1 Study Outline. GROC, participant Global Rating of Change; DASH, Disability of the Arm, Shoulder and Hand; PFSF, Patient Specific Functional Scale; S-LANSS, Self-reported-Leeds Assessment of Neuropathic Symptoms and Signs.

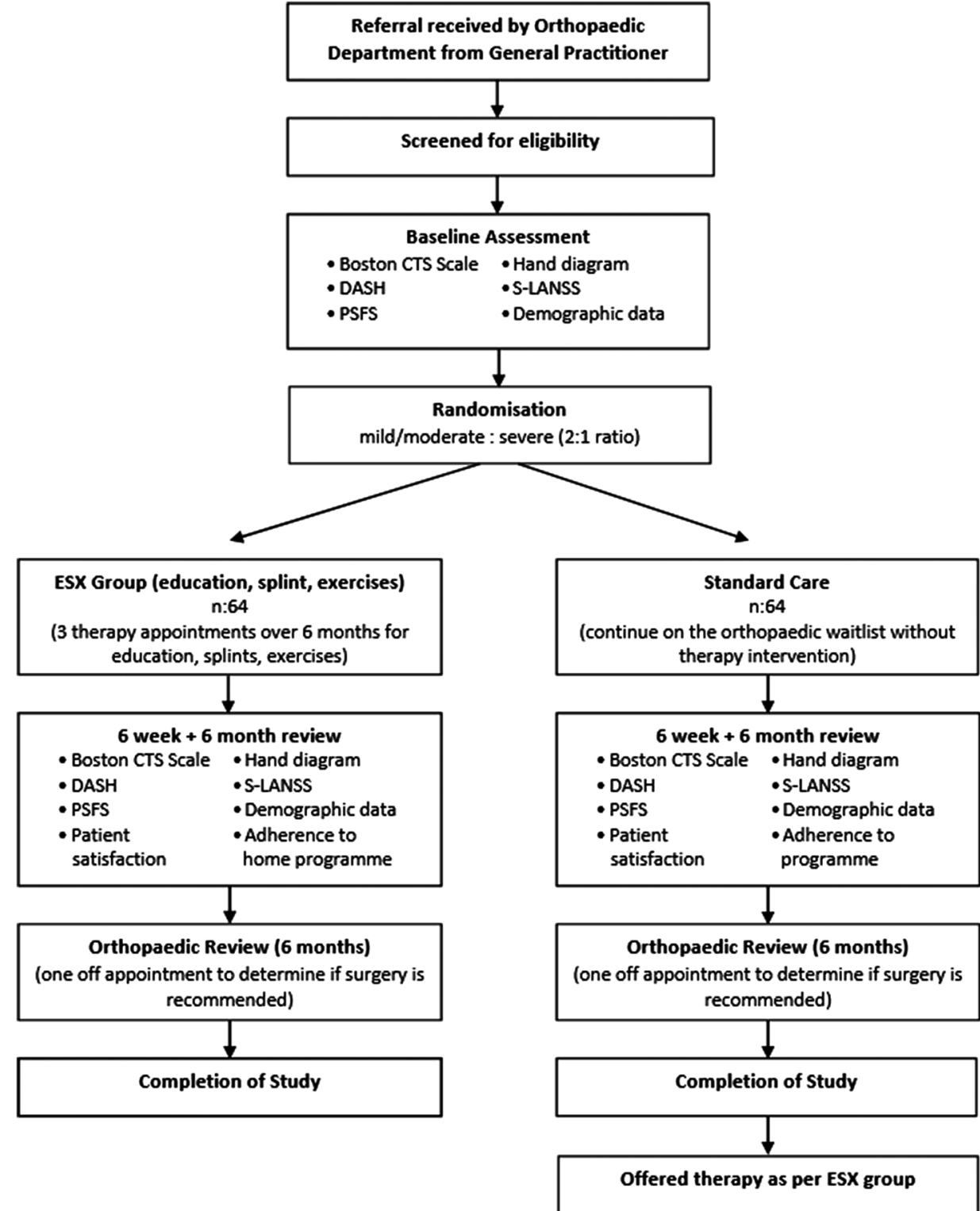

supports with custom moulded thermoplastic stays that hold the wrist in a neutral position (figure 2A). Those with a positive Berger test will receive splints as described above but that extend distally to the level of the proximal phalanx and therefore limit metacarpal phalangeal joint flexion (figure 2B). Splinting has been shown to be of benefit in reducing symptoms ${ }^{9}{ }^{14}$ and patients will be requested to wear the splint during the night only. $^{27} 28$

In addition to education and splinting, participants will be advised on a home exercise programme consisting of four exercises including median nerve and tendon-gliding exercises (figure 3A-C). ${ }^{28-32}$ During the education session, therapists will assure that an accurate performance of these exercises is achieved by the patients. Nerve and tendon-gliding exercises have been shown to have a positive impact on symptoms ${ }^{14}$ and reduce intraneural oedema in patients with CTS. $^{28}$ Patients are asked to perform 5-10 repetitions of each exercise five times a day in a manner that does not cause pain or increase symptoms. Participants who report an exercise-related increase of symptoms at any point during the study will be advised to contact the occupational therapist or physiotherapist. On doing so, the participant will be asked to trial a slightly modified version (such as completing exercises through a reduced range of motion) or to cease exercises completely if necessary.

\section{Standard care}

The standard care group will continue as per current practice to remain on the orthopaedic department outpatient waitlist for the length of the study (6 months) without receiving the education, splint or exercises described above. At the completion of the trial, the participants who underwent standard care (and have not had surgery) will be offered the option to receive the ESX intervention outlined above. 


\section{Box 1 Participant selection criteria}

\section{Inclusion criteria}

- Referral to participating hospitals' orthopaedic department with a diagnosis of carpal tunnel syndrome (CTS);

- Diagnosis of CTS confirmed by nerve conduction studies;

- Clinical symptoms and signs consistent with CTS, such as altered sensation, numbness, paraesthesia or pain within the affected hand;

- Symptoms longer than 2 months;

- 18-75 years of age;

- Ability to comprehend the study, its requirements and provide consent.

Exclusion criteria

- Pregnancy-related CTS;

- Systemic disease other than diabetes;

- Osteoarthritis of the wrist or hand;

- Musculoskeletal conditions affecting the elbow, hand and wrist (such as de Quervain's tenosynovitis or trigger finger);

- Traumatic onset of CTS;

- Neurological conditions affecting the upper limb;

- Use of hand therapy interventions within the previous 3 months (splints or exercises);

- Steroid injection for CTS within the previous 6 months;

Pending litigation or insurance claim.

\section{Randomisation and allocation}

The randomisation schedule will be generated using the Research Randomizer software (GC Urbaniak, S Plous. Research Randomizer (Version 4.0). Computer software. http://www.randomizer.org/2013 accessed 23 Sept 2013) and administered by an investigator who will not be involved with participant assessment, allocation or treatment. Allocation will be completed following consent and baseline assessment using sealed envelopes. Researchers completing the analysis and therapists completing the outcome measures will be blinded to allocation. Participants will be randomised into either mild/

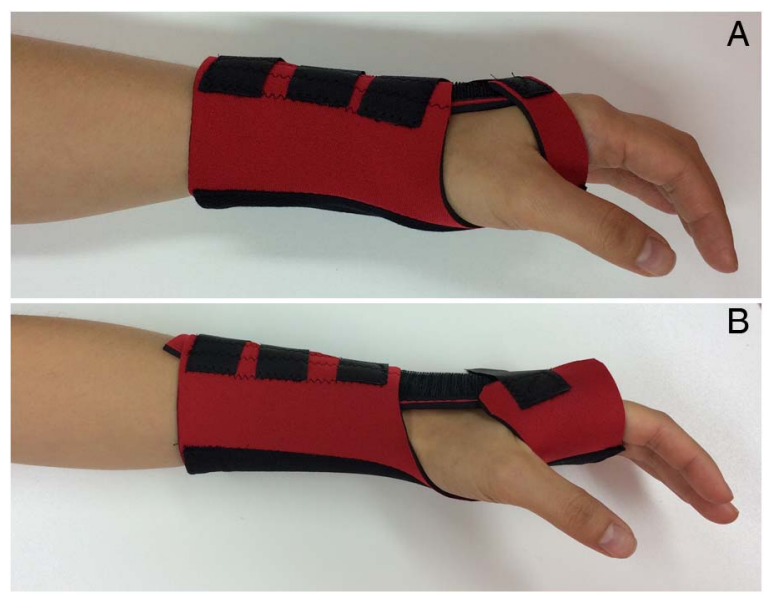

Figure 2 (A) Night splint-wrist included in neutral position (used if participant has a negative Berger's test). (B) Night splint-wrist and Metacarpal phalangeal joints included in neutral position (used if participant has a positive Berger's test). moderate or severe groups at a ratio of 2:1. For those with bilateral symptoms, the hand chosen to be included in the study will be allocated at random by flipping a coin, and both hands will be managed as per group allocation of the included hand.

\section{Stratification}

Since it is expected that those with severe CTS will be less likely to respond to therapy, allocation will be stratified into mild/moderate or severe according to neurophysiological test severity according to the classification as suggested by Bland. ${ }^{33}$ Severe CTS is defined as a score of 4 or above (severe, very severe and extremely severe, respectively) on the Bland classification.

\section{Outcome measures}

A battery of outcome measures will be used with the primary outcome measures being 'conversion to surgery' and the participant global rating of change. An investigator who is blinded to group allocation will administer the outcome measures at baseline and at 6 weeks and 6 months after enrolment in the study. The only exceptions to these time frames are "conversion to surgery' which will only be evaluated at 6 months by an orthopaedic surgeon, and the participant global rating of change which will be evaluated at 6 weeks and 6 months.

\section{Primary outcome measures \\ Conversion to surgery}

Conversion to surgery is based on the surgeon's recommendation regarding whether or not the participant should have surgery. This decision will be made during a face-to-face appointment (as per standard practice) with the participant and an orthopaedic surgeon (either a Registrar or Consultant) at 6 months following randomisation, or earlier if rapid deterioration is identified. During this appointment, the surgeon will complete a form, which asks whether or not they would recommend surgery for that patient given their presentation and symptom severity at the appointment. Those identified as requiring surgery will be booked for carpal tunnel release as per standard practice at participating public hospitals. Those deemed to not require surgery will be discharged back into the care of their general medical practitioner. The percentage of participants requiring a carpal tunnel release in both groups will be compared. We will also seek participants' perspective whether they wish to proceed to surgery prior to their appointment with the surgeons. Comparison of conversion to surgery has been used in previous clinical trials to determine the success of non-surgical management. ${ }^{34}$

\section{Participant Global Rating of Change}

Participants will be asked to rate the change in their symptoms since starting the study on the global rating of change scale. ${ }^{35}$ This scale measures perceived improvement on a 15-point Likert scale ranging from a very great deal worse 
Figure 3 (A-C) Exercises performed by the ESX group (A) Median nerve-gliding exercises (forearm) ${ }^{29}$ (B) median nerve-gliding exercises (wrist and fingers) and (C) tendon-gliding exercises $^{30} \mathrm{ESX}$, education, splinting and excercise. All exercises will be completed with $5-10$ repetitions, 5 times per day in a pain-free manner.
A

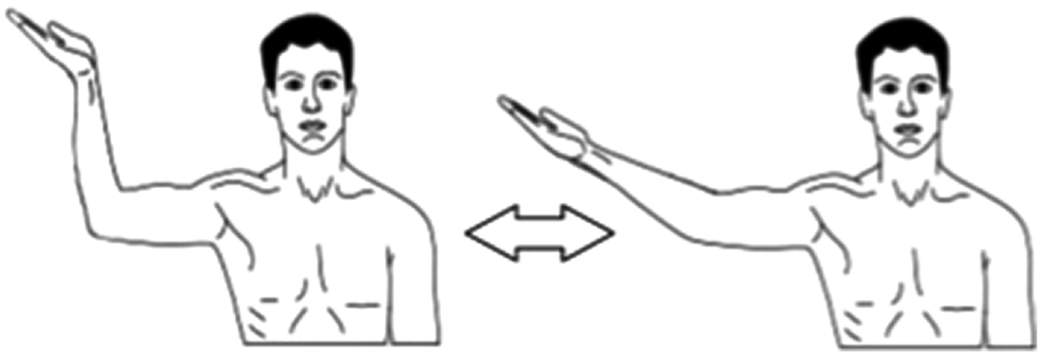

B

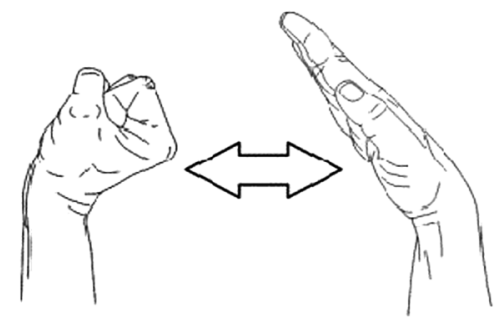

C

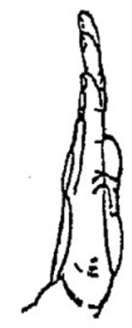

to a very great deal better. ${ }^{36}$ This questionnaire has previously been shown to have good reliability and validity in patients with musculoskeletal disorders. ${ }^{37}$ As previously described, a score of $\geq 5$ points (at least a 'good deal better') on this scale will be classified as 'improved'. 38 39

\section{Secondary outcome measures}

\section{Boston CTS Questionnaire}

The Boston CTS Questionnaire is a two component, selfadministered questionnaire. This questionnaire is CTS diagnosis specific, reliable, valid and responsive to change. ${ }^{40}$ Both subscales result in a score between 1 (least severe) and 5 (most severe).

\section{Disability of the Arm, Shoulder and Hand}

The Disability of Arm, Shoulder and Hand (DASH) is a self-administered questionnaire that is responsive, ${ }^{42}$ reliable and valid in patients with upper limb disorders ${ }^{43}$ and CTS. ${ }^{44} 45$ It comprises 30 questions with components relating to symptoms, functional status ${ }^{46}$ and hand use. The final score ranges from 0 to 100 and a 10-point change in the total DASH score has been suggested as the minimal clinically relevant difference in repeat scores following carpal tunnel release. ${ }^{47}$

\section{Participant completed hand and body diagram representing symptom distribution}

Participants will be asked to mark symptom type and location on a diagram in order to monitor symptom distribution. This outcome measure is commonly used in patients with $\mathrm{CTS}^{48}$ and will be used to evaluate symptom spread and the presence of extra-median symptoms. ${ }^{49} 50$ Self-completed hand diagrams have been shown to be reliable in patients with CTS. ${ }^{51}$

\section{The Patient-Specific Functional Scale}

At baseline, participants will be asked to identify three important tasks that they are unable to do or have difficulty doing as a result of their hand problem. They will rate the difficulty they experience in completing that activity on a scale ranging from 0 (unable to perform the activity) to 10 (able to perform the activity at the same level as before the injury or problem). This scale has been established as reliable, valid and responsive in patients with musculoskeletal conditions ${ }^{52}$ and upper extremity nerve injuries. ${ }^{53}$ At the 6-week and 6-month time points, the patients will repeat the rating of the same activities on the 11-point scale. ${ }^{54}$

\section{Self-reported Leeds Assessment of Neuropathic Symptoms and Signs}

The Self-reported Leeds Assessment of Neuropathic Symptoms and Signs (S-LANSS) scale is a seven question self-reported scale that aims to identify pain of neuropathic origin. ${ }^{55}$ A value of $\geq 12$ points on this scale is considered to be indicative of neuropathic pain. This questionnaire has been shown to be valid and reliable in 
patients with neuropathic pain ${ }^{55}$ and has previously been used in patients with CTS. ${ }^{56}$

\section{Patient satisfaction with the treatment/management process}

Since this study represents a change in care practices, patient satisfaction with the received treatment process will be included. The outcome measure to be used was adapted from Hall $e t a \bar{p}^{7}$ and asks patients to rate their perceptions of treatment, satisfaction, function and progress on a seven question, 10-point Likert scale (see online supplementary appendix 1 ).

\section{Adherence to home programme}

Treating therapists will retrospectively monitor adherence to the above therapy programme at the 6-week and 6-month review appointments. ${ }^{58}$ Adherence will be documented on a standardised form which details regularity of exercise completion and splint use, and if exercises could be correctly demonstrated to the therapist, they will also be recorded. Any deviations from the protocol, such as the receipt of any additional therapy or interventions for CTS, will be recorded; however, participants will continue within the study pathway as per randomisation.

\section{Participant monitoring and management of adverse events}

Following the 6-week review appointment, a therapist will discuss the progress of all participants (both standard care and ESX groups) with an orthopaedic consultant in the form of a brief case conference. This case conference will be used to provide an update regarding patient progress and, in particular, identify any participants with rapidly deteriorating symptoms for whom an orthopaedic review prior to the completion of the study may be indicated.

Participants will be encouraged to contact their treating therapist between appointments if any concerns arise regarding their home programme or if they experience an increase in symptoms. These concerns will be addressed by their treating therapist and details of the issue and outcome recorded. Any adverse events will be recorded and reported to the ethics committee as per institutional ethics committee requirements.

\section{Training of therapists}

All therapists involved in treating or assessing study participants will have completed training in regard to interventions and procedures. This training will include a self-directed review of the research protocol and reading three textbook chapters relating to contemporary rehabilitation of CTS. ${ }^{5} 5960$ To maintain consistency of practice, therapists will observe a member of the research team complete a clinical examination and ESX interventions and they will also complete these processes under guidance. Senior therapists and a site coordinator will be available to provide guidance in relation to the implementation of the research protocol and provide clinical assistance as needed.

\section{Trial management}

Data will be collected, managed, stored and confidentiality maintained as per Queensland Health policies. Investigators will meet regularly to monitor and discuss trial conduct. Additionally, this trial is subject to random audit by the approving research ethics committee. Protocol amendments will first be approved by the ethics committee and then disseminated to site investigators via meetings and updating of study resources and guidelines. All authors will have access to the final trial data set.

\section{Sample size}

The primary aim of this study is to detect a clinically important difference between the standard care and ESX groups in conversion to surgery rates. Power calculation revealed that 64 patients (stratified according to electrodiagnostic test severity into $43 \mathrm{mild} /$ moderate, 21 severe) are required per group to detect a 25\% lower conversion to surgery, assuming that the usual conversion rate is $69 \%^{20}$ with a power of $80 \%$ at a $95 \%$ confidence level and allowing for a $5 \%$ loss to follow-up rate.

\section{Planned data analysis}

Appropriate descriptive statistics for all outcome measures and demographic characteristics across groups will be reported for baseline, 6 weeks and 6 months. Demographic and outcome measurement data at baseline will be assessed for comparability between groups. Comparative analyses between treatments will be performed using an intention-to-treat approach by an investigator blind to allocation. Outcomes will be analysed using linear mixed or logistic regression models, including respective baseline scores as a covariate, participants as a random effect, treatments as a fixed factor and covariate by treatment interaction at 6 weeks and 6 months. Regression diagnostics will be used to test data fit to assumptions. Hypotheses will be statistically tested at the $95 \%$ confidence limits.

\section{DISCUSSION}

Public health services are faced with the challenge of improving efficiency and managing increasing patient numbers with limited staffing and financial resources. ${ }^{61}$ An ageing population and increased complexity of healthcare needs driven by the burden of chronic disease create significant challenges in provision of healthcare services. ${ }^{62}$ Long waitlists for specialist orthopaedic appointments and surgery has been identified as a global issue. ${ }^{6163-65}$ This project aims to investigate the efficacy of an alternative care pathway that may assist in streamlining care and improve outcomes for patients with CTS who are on surgical waitlists.

Owing to limited clinical evidence for the non-surgical management of CTS, the ESX interventions were based on trends in clinical care and the success of similar clinical care models in retrospective reports. The choice of exercises followed evidence from previous studies which suggested beneficial biomechanical and neurophysiological 
effects of these exercises in patients with CTS. ${ }^{28} 29$ The relative ease of exercise completion and splint use may encourage patient self-management, thus limiting the number of therapy appointments required.

It has been suggested that conservative management for those with severe CTS is unlikely to reduce the need for surgery. ${ }^{6}$ However, there is limited evidence relating to whether conservative management offers other benefits to those with severe symptoms, even if surgery is the end result. This information could be of significance for both patients and those managing their care. To assist in answering this question, participants with severe CTS will not be excluded from the study, but their group allocation will be stratified (mild/moderate and severe), allowing subgroup analysis. It is acknowledged that those with severe CTS are recommended to obtain care within a timely manner in order to prevent exacerbation of symptoms. In an effort to reduce this risk, the study follow-up time of 6 months is significantly less than current wait times (between 9 months and 6 years at the time of study commencement) to access specialist care at the sites included within this study. Additionally, participants will be monitored and managed as discussed earlier in this manuscript.

The surgeons' opinion on whether surgery is or is not required was chosen as the basis for the conversion to surgery outcome measures. Surgeons are the primary decision-makers in determining the need for surgery in the majority of settings. We will seek participants' perspective whether they wish to proceed to surgery prior to their appointment with the surgeons. This will allow evaluation of the relationship between surgeon and patient perspective in regard to need for surgery.

Since the care pathway being investigated uses clinically accessible interventions and outcome measures, it is anticipated that the results of this study will be applicable to the management of CTS in a wide range of hospital settings.

\section{CONCLUSION}

This paper outlines the design and rationale for a randomised controlled trial that aims to assess the effectiveness of an intervention clinic for the management of patients with CTS on the surgery waitlist. It is anticipated that the outcomes of this study will contribute to an improved and expedited management of this common condition in a clinical setting.

\footnotetext{
Author affiliations

${ }^{1}$ Occupational Therapy Department, Gold Coast University Hospital, Queensland, Australia

${ }^{2}$ School of Health and Rehabilitation Sciences, The University of Queensland, Brisbane, Queensland, Australia

${ }^{3}$ Occupational Therapy Department, Queen Elizabeth II Jubilee Hospital, Brisbane, Queensland, Australia

${ }^{4}$ MOVE Research Institute Amsterdam, Department of Human Movement Sciences, Faculty of Behavioural and Movement Sciences, Vrije Universiteit Amsterdam, Amsterdam, The Netherlands

${ }^{5}$ Nuffield Department of Clinical Neurosciences, University of Oxford, England, UK
}

Twitter Follow Bill Vicenzino at @Bill_Vicenzino Follow Annina Schmid @AnninaBSchmid

Acknowledgements The authors would like to thank Dr Tyson Doneley and Mr Michael Tay at QEII Jubilee Hospital for their support in developing this clinic model. Acknowledgements are due to QEII Jubilee, Rockhampton, Gold Coast University Hospital and Logan Hospital Occupational Therapy, Physiotherapy and Orthopaedic Departments for their site participation.

Contributors KJL is the primary investigator. KJL, ABS, LR, MWC and BV participated in the development of the study design and conduct. KJL, ABS and LR contributed to applying for and gaining funding. All authors contributed to the content and critical revision and approved the final draft of the manuscript.

Funding $A B S$ is funded by a Neil Hamilton Fairley Fellowship from the National Health and Medical Research Council Australia. This study is supported in part by a Health Practitioners Stimulus Grant from Queensland Health.

\section{Competing interests None declared.}

Ethics approval Ethics approval was gained via the Princess Alexandra Hospital Centres for Health Research (HREC/13/QPAH/434-SSA/13/QPAH/ 447) and the Medical Research Ethics Committee at the University of Queensland.

Provenance and peer review Not commissioned; externally peer reviewed.

Open Access This is an Open Access article distributed in accordance with the Creative Commons Attribution Non Commercial (CC BY-NC 4.0) license, which permits others to distribute, remix, adapt, build upon this work noncommercially, and license their derivative works on different terms, provided the original work is properly cited and the use is non-commercial. See: http:// creativecommons.org/licenses/by-nc/4.0/

\section{REFERENCES}

1. Bland JD. Carpal tunnel syndrome. Curr Opin Neurol 2005;18:581-5.

2. Diagnosis of the carpal tunnel syndrome. Lancet 1985;1:854-5

3. Atroshi I, Gummesson C, Johnsson R, et al. Prevalence of carpal tunnel syndrome in a general population. JAMA 1999;282:153-8.

4. Dale AM, Harris-Adamson C, Rempel D, et al. Prevalence and incidence of carpal tunnel syndrome in US working populations: pooled analysis of six prospective studies. Scand J Work Environ Health 2013;39:495-505.

5. Evans R. Therapist's management of carpal tunnel syndrome: a practical approach. In: Skirven TM, Fedorczyk JM, Amadio PC. In rehabilitaiton of the hand and upper extremity, O.A. eds. Elsevier, 2011:666-77.

6. Keith MW, Masear V, Chung KC, et al. American academy of orthopaedic surgeons: clinical practice guideline on the treatment of carpal tunnel syndrome. J Bone Joint Surg Am 2010;92:218-19.

7. Foley MB, Silverstein B, Polissar N. The economic burden of carpal tunnel syndrome: long-term earnings of CTS claimants in Washington State. Am J Ind Med 2007;50:155-72.

8. Verdugo RJ, Salinas RA, Castillo JL, et al. Surgical versus non-surgical treatment for carpal tunnel syndrome. Cochrane Database Syst Rev 2008(4):CD001552.

9. Shi Q, MacDermid JC. Is surgical intervention more effective than non-surgical treatment for carpal tunnel syndrome? A systematic review. J Orthop Surg Res 2011;6:17.

10. Jerosch-Herold C, Shepstone L, Wilson EC, et al. Clinical course, costs and predictive factors for response to treatment in carpal tunnel syndrome: the PALMS study protocol. BMC Musculoskelet Disord 2014;15:35.

11. Atroshi I, Gummesson C, Johnsson R, et al. Symptoms, disability, and quality of life in patients with carpal tunnel syndrome. $J$ Hand Surg Am 1999;24:398-404.

12. Chandra PS, Singh PK, Goyal V, et al. Early versus delayed endoscopic surgery for carpal tunnel syndrome: prospective randomized study. World Neurosurg 2013;79:767-72.

13. American Association of Neuromuscular and Electrodiagnostic Medicine: practice guidelines endorsed by the AANEM. http://www. aanem.org/Practice/Practice-Guidelines.aspx (accessed 19 Mar 2015). 
14. Huisstede BM, Hoogvliet $\mathrm{P}$, Randsdorp MS, et al. Carpal tunnel syndrome. Part I: effectiveness of nonsurgical treatments-a systematic review. Arch Phys Med Rehabil 2010;91:981-1004.

15. Page MJ, Massy-Westropp N, O'Connor D, et al. Splinting for carpal tunnel syndrome. Cochrane Database Syst Rev 2012;(7):CD010003.

16. Page MJ, O'Connor D, Pitt $\mathrm{V}$, et al. Exercise and mobilisation interventions for carpal tunnel syndrome. Cochrane Database Syst Rev 2012;6:CD009899.

17. Bebbington E, Furniss D. Linear regression analysis of Hospital Episode Statistics predicts a large incresae in demand for elective hand surgery in England. $J$ Plast Reconstr Aesthet Surg 2015;68:243-51.

18. National Tariff Payment System 2014/15: Annex 5A National Prices. Accessed 7.10.14.

19. The Statement of Health Service Treatment Costs Northern Ireland 2013-14. http://www.dhsspsni.gov.uk/the_statement_of_health_ care treatment costs northern ireland 2013 14.pdf

20. Storey PA, Dear H, Bradley MJ, et al. Audit of a therapist-led clinic for carpal tunnel syndrome in primary care. $\mathrm{Br} J$ Hand Ther 2008;13:72-8.

21. Warwick D, Belward P. Hand Therapist Carpal Tunnel Clinic. Br J Hand Ther 2004;9:23-6.

22. O'Brien L, Hardman A, Goldby S. The impact of a hand therapy screening and management clinic for patients referred for surgical opinion in an Australian public hospital. $J$ Hand Ther 2013;26:318-22; quiz 322

23. Viikari-Juntura E, Silverstein B. Role of physical load factors in carpal tunnel syndrome. Scand J Work Environ Health 1999;25:163-85.

24. Siegel DB, Kuzma G, Eakins D. Anatomic investigation of the role of the lumbrical muscles in carpal tunnel syndrome. J Hand Surg Am 1995;20:860-3.

25. Yii NW, Elliot D. A study of the dynamic relationship of the lumbrical muscles and the carpal tunnel. J Hand Surg Br 1994;19: 439-43.

26. Cobb TK, An KN, Cooney WP, et al. Lumbrical muscle incursion into the carpal tunnel during finger flexion. J Hand Surg Br 1994;19:434-8.

27. Brininger TL, Rogers JC, Holm MB, et al. Efficacy of a fabricated customized splint and tendon and nerve gliding exercises for the treatment of carpal tunnel syndrome: a randomized controlled trial. Arch Phys Med Rehabil 2007;88:1429-35.

28. Schmid AB, Elliott JM, Strudwick MW, et al. Effect of splinting and exercise on intraneural edema of the median nerve in carpal tunnel syndrome-an MRI study to reveal therapeutic mechanisms. J Orthop Res 2012;30:1343-50.

29. Coppieters MW, Alshami AM. Longitudinal excursion and strain in the median nerve during novel nerve gliding exercises for carpal tunnel syndrome. J Orthop Res 2007:25:972-80.

30. Palmer A. Fractures of the Distal Radius. In: Operative Hand Surgery 3rd Edition. Edited by DP G. New York : Churchill Livingston, 1993:943.

31. Horng YS, Hsieh SF, Tu YK, et al. The comparative effectiveness of tendon and nerve gliding exercises in patients with carpal tunnel syndrome: a randomized trial. Am J Phys Med Rehabil 2011;90:435-42.

32. Horng YS, Hsieh SF, Lin MC, et al. Ultrasonographic median nerve changes under tendon gliding exercise in patients with carpal tunnel syndrome and healthy controls. J Hand Ther 2014;27:317-23.

33. Bland JD. A neurophysiological grading scale for carpal tunnel syndrome. Muscle Nerve 2000;23:1280-3.

34. Napier C, McCormack RG, Hunt MA, et al. A physiotherapy triage service for orthopaedic surgery: an effective strategy for reducing wait times. Physiother Can 2013;65:358-63.

35. Kamper SJ, Maher CG, Mackay G. Global rating of change scales: a review of strengths and weaknesses and considerations for design. J Man Manip Ther 2009;17:163-70.

36. Lauridsen $\mathrm{HH}$, Hartvigsen J, Korsholm L, et al. Choice of external criteria in back pain research: does it matter? Recommendations based on analysis of responsiveness. Pain 2007;131:112-20.

37. Kamper SJ, Ostelo RW, Knol DL, et al. Global Perceived Effect scales provided reliable assessments of health transition in people with musculoskeletal disorders, but ratings are strongly influenced by current status. J Clin Epidemiol 2010;63:760-6 e1.

38. Stratford PW, Binkley J, Solomon P, et al. Assessing change over time in patients with low back pain. Phys Ther 1994;74:528-33.

39. Perillo M, Bulbulian R. Responsiveness of the Bournemouth and Oswestry questionnaires: a prospective pilot study. J Manipulative Physiol Ther 2003;26:77-86.

40. Leite JC, Jerosch-Herold C, Song F. A systematic review of the psychometric properties of the Boston Carpal Tunnel Questionnaire. BMC Musculoskelet Disord 2006;7:78.
41. Levine DW, Simmons BP, Koris MJ, et al. A self-administered questionnaire for the assessment of severity of symptoms and functional status in carpal tunnel syndrome. $J$ Bone Joint Surg Am 1993; 75:1585-92

42. Gay RE, Amadio PC, Johnson JC. Comparative responsiveness of the disabilities of the arm, shoulder, and hand, the carpal tunnel questionnaire, and the SF-36 to clinical change after carpal tunnel release. J Hand Surg Am 2003;28:250-4.

43. Beaton DE, Katz JN, Fossel AH, et al. Measuring the whole or the parts? Validity, reliability, and responsiveness of the Disabilities of the Arm, Shoulder and Hand outcome measure in different regions of the upper extremity. $J$ Hand Ther 2001;14:128-46.

44. Hobby JL, Watts $\mathrm{C}$, Elliot $\mathrm{D}$. Validity and responsiveness of the patient evaluation measure as an outcome measure for carpal tunnel syndrome. J Hand Surg Br 2005;30:350-4.

45. Sambandam SN, Priyanka P, Gul A, et al. Critical analysis of outcome measures used in the assessment of carpal tunnel syndrome. Int Orthop 2008;32:497-504.

46. Hudak PL, Amadio PC, Bombardier C. Development of an upper extremity outcome measure: the DASH (disabilities of the arm, shoulder and hand) [corrected]. The Upper Extremity Collaborative Group (UECG). Am J Ind Med 1996;29:602-8.

47. Sorensen AA, Howard D, Tan $\mathrm{WH}$, et al. Minimal clinically important differences of 3 patient-rated outcomes instruments. J Hand Surg Am 2013;38:641-9.

48. Katz JN, Larson MG, Sabra A, et al. The carpal tunnel syndrome: diagnostic utility of the history and physical examination findings. Ann Intern Med 1990;112:321-7.

49. Zanette G, Marani S, Tamburin S. Extra-median spread of sensory symptoms in carpal tunnel syndrome suggests the presence of pain-related mechanisms. Pain 2006;122: 264-70.

50. Zanette G, Cacciatori C, Tamburin S. Central sensitization in carpal tunnel syndrome with extraterritorial spread of sensory symptoms. Pain 2010;148:227-36.

51. Dale AM, Strickland J, Symanzik J, et al. Reliability of hand diagrams for the epidemiologic case definition of carpal tunnel syndrome. J Occup Rehabil 2008;18:233-48.

52. Abbott JH, Schmitt JS. The Patient-Specific Functional Scale was valid for group-level change comparisons and between-group discrimination. J Clin Epidemiol 2014;67:681-8.

53. Novak CB, Anastakis DJ, Beaton DE, et al. Validity of the Patient Specific Functional Scale in patients following upper extremity nerve injury. Hand (N Y) 2013;8:132-8.

54. Hefford $\mathrm{C}$, Abbott $\mathrm{JH}$, Arnold $\mathrm{R}$, et al. The patient-specific functional scale: validity, reliability, and responsiveness in patients with upper extremity musculoskeletal problems. J Orthop Sports Phys Ther 2012:42:56-65.

55. Bennett MI, Smith BH, Torrance N, et al. The S-LANSS score for identifying pain of predominantly neuropathic origin: validation for use in clinical and postal research. J Pain 2005;6:149-58.

56. Gürsoy AE, Kolukısa M, Yıldız GB, et al. Relationship between electrodiagnostic severity and neuropathic pain assessed by the LANSS pain scale in carpal tunnel syndrome. Neuropsychiatr Dis Treat 2013;9:65-71.

57. Hall B, Lee HC, Fitzgerald $\mathrm{H}$, et al. Investigating the effectiveness of full-time wrist splinting and education in the treatment of carpal tunnel syndrome: a randomized controlled trial. Am J Occup Ther 2013;67:448-59.

58. Bassett SF. The assessment of patient adherence to physiotherapy rehabilitation. New Zealand J Physiother 2003;31:60-6.

59. Jacoby SM, EM Osterman AL. Basic science of nerve compression. In: Skirven TM, Fedorczyk JM, Amadio PC. In rehabilitation of the hand and upper extremity, O.A. eds. Elsevier, 2011:649-56.

60. Amadio P. Carpal tunnel syndrome: surgeon's management. In: In Rehabilitation of the Hand and Upper Extremity, O.A. Skirven TM, Fedorczyk JM, Amadio PC. eds. Elsevier, 2011:657-65.

61. Radnor ZJ, Holweg M, et al. Lean in healthcare: the unfilled promise? Soc Sci Med 2012;74:364-71.

62. Litvak E, Bisognano M. More patients, less payment: increasing hospital efficiency in the aftermath of health reform. Health Aff (Millwood) 2011;30:76-80.

63. Mascarenhas R. The Manitoba arthroplasty waiting list: impact on health-related quality of life and initiatives to remedy the problem. $J$ Eval Clin Pract 2009:15:208-11.

64. De Coster C, McMillan S, Brant R, et al. The Western Canada Waiting List Project: development of a priority referral score for hip and knee arthroplasty. J Eval Clin Pract 2007;13:192-7.

65. Hacker J, Stanistreet D. Equity in waiting times for two surgical specialties: a case study at a hospital in the North West of England. $J$ Public Health (Oxf) 2004;26:56-60. 